\title{
Remote surface damage detection on rotor blades of operating wind turbines by means of infrared thermography
}

\author{
Dominik Traphan ${ }^{1}$, Iván Herráez ${ }^{2}$, Peter Meinlschmidt ${ }^{3}$, Friedrich Schlüter ${ }^{3}$, Joachim Peinke ${ }^{1}$, and \\ Gerd Gülker ${ }^{1}$ \\ ${ }^{1}$ ForWind, Institute of Physics, University of Oldenburg, Oldenburg, Germany \\ ${ }^{2}$ Department of Technology, University of Applied Sciences Emden/Leer, Emden, Germany \\ ${ }^{3}$ Fraunhofer Institute for Wood Research, Wilhelm-Klauditz-Institut WKI, Braunschweig, Germany
}

Correspondence: Dominik Traphan (dominik.traphan@uni-oldenburg.de)

Received: 5 March 2018 - Discussion started: 3 April 2018

Revised: 30 July 2018 - Accepted: 19 August 2018 - Published: 13 September 2018

\begin{abstract}
Wind turbines are constantly exposed to wind gusts, dirt particles and precipitation. Depending on the site, surface defects on rotor blades emerge from the first day of operation on. While erosion increases quickly with time, even small surface defects can affect the performance of the wind turbine. Consequently, there is demand for an easily applicable remote monitoring method for rotor blades that is capable of detecting surface defects at an early stage. In this work it is investigated if infrared thermography (IRT) can meet these requirements by visualizing differences in the thermal transport and the corresponding surface temperature of the wall-bounded flow.

Firstly, a validation of the IRT method compared to stereoscopic particle image velocimetry measurements is performed comparing both types of experimental results for the boundary layer of a flat plate. Then, the main characteristics of the flow in the wake of generic surface defects on different types of lifting surfaces are studied both experimentally and numerically: temperature gradients behind protruding surface defects on a flat plate and a DU 91-W2-250 profile are studied by means of IRT. The same is done with the wall shear stress from Reynolds-averaged Navier-Stokes simulations of a wind turbine blade. It is consistently observed, both in the experiments and the simulations, that turbulent wedges are formed on the flow downstream of generic surface defects. These wedges provide valuable information about the kind of defects that generate them. At last, experimental and numerical performance measures are taken into account for evaluating the aerodynamic impact of surface defects on rotor blades. We conclude that the IRT method is a suitable remote monitoring technique for detecting surface defects on wind turbines at an early stage.
\end{abstract}

\section{Introduction}

Wind turbines operate under transient flow conditions in the harsh environment of the atmospheric boundary layer (BL) both on- and offshore. Directly exposed to heavy wind gusts, dirt particles or precipitation, rotor blades suffer from fatigue, erosion and contamination (cf. Ghoshal et al., 2000; Soltani et al., 2011; Sareen et al., 2014). Emerging damages range from initial surface defects to structural threats. In avoidance of rotor blade breakdown, a variety of re- mote damage detection methods have been developed, some of which are widely used in practice (Ciang et al., 2008; Márquez et al., 2012; Li et al., 2015). Most methods are based on measuring a global effect by local sensors (e.g., acoustic emission), thus lacking in detectability of small damages and their exact localization. More accurate methods, such as scanning laser Doppler vibrometers, are not practical due to complex application and bulky equipment (Liu et al., 2015). Another common method is a visual inspection to find surface defects, but stopping the turbine and 
climbing along rotor blades is very costly and time consuming, particularly offshore.

It is well known across industry and science that even small surface inhomogeneities on rotor blades (erosion, contamination) can cause significant power loss and unfavorable dynamic loads (Corten and Veldkamp, 2001; Timmer and Schaffarczyk, 2004; Soltani et al., 2011; Torres-Nieves et al., 2012; Ehrmann et al., 2013; Bak et al., 2016). However, the impact of small surface defects on performance of airfoils and rotor blades has not yet been quantified systematically. Once an initial defect emerges, erosion progresses quickly. Thus, even small surface defects need to be detected already at an early stage if more severe damage is to be prevented.

Based on the arguments mentioned above, there is a demand for a remotely operated and easily applicable inspection method of rotor blades which is able to identify even small surface defects. Infrared thermography (IRT) has the potential to meet these requirements. It is a non-intrusive imaging technique that visualizes the wall-bounded flow by resultant temperature gradients. Due to heat transfer between a rotor blade and free flow, such gradients appear when the blade and surrounding air differ in temperature. Depending on whether the flow is warmer or colder than the rotor blade, turbulent regions appear warmer or colder on the surface as they apply a higher convective heating or cooling. In the most general sense, every surface property induces a flow feature which implies a certain convective cooling or heating, hence being potentially recognizable in a thermogram. In visualization of wall-bounded flows, IRT provides a spatial accuracy in the subpixel range which is in practice limited by remaining unsteadiness of the flow itself (Dollinger et al., 2018b). More details about the underlying physical theory of IRT and a selection of applications are given by Carlomagno and Cardone (2010).

In general, single surface roughness elements generating so-called turbulent wedges in the BL were the subject of several basic studies. Thermographic flow visualization based on chemical surface treatment for the purpose of defect identification lacks in practicability or spatial resolution (Zhong et al., 2003; Kuester and White, 2016). Other studies investigated roughness elements that significantly exceed typical dimensions encountered on rotor blades (Rudolph et al., 2009). On airfoils and helicopter rotor blades, turbulent wedges were visualized by IRT without in-depth analysis (Horstmann et al., 1990; Kucaba-Piętal et al., 2013; Richter and Schülein, 2014).

In the field of wind turbine maintenance, IRT is usually not considered a suitable inspection method on site up to now. According to some studies, the application of IRT requires thermal excitation to enhance thermal contrast, which is very laborious (Ciang et al., 2008; Márquez et al., 2012; Li et al., 2015). However, the latest development of infrared cameras yielded compact cameras with a thermal resolution of better than $15 \mathrm{mK}$ (Carlomagno and Cardone, 2010; Rogalski, 2011). In this way, infrared cameras are able to ex-

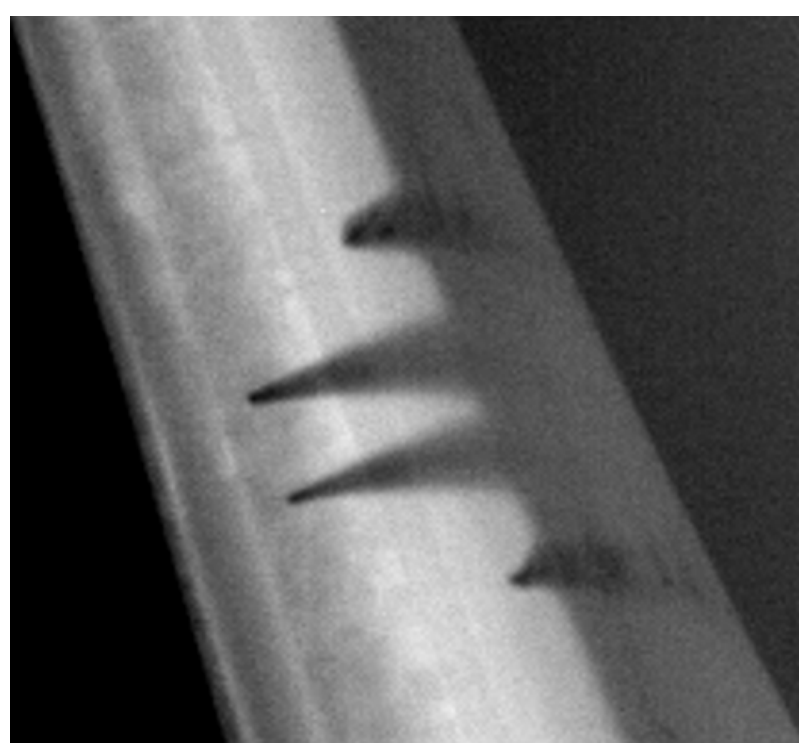

Figure 1. Thermogram of a rotor blade's suction side of an operating wind turbine: natural laminar-turbulent boundary layer (BL) transition and turbulent wedges generated by surface roughness elements appear darker. The view is from behind the turbine; i.e., movement is leftwards. The thermogram was taken with a $200 \mathrm{~mm}$ telephoto lens in the morning sun; i.e., surface is about $2{ }^{\circ} \mathrm{C}$ colder than the air.

ploit naturally occurring temperature differences to image the whole rotor blade at once as recently proposed for distinguishing flow regimes on rotor blades of operating wind turbines (Dollinger et al., 2018a).

A thermogram of a moving rotor blade after just a couple of months of operation is depicted in Fig. 1 (color coded in gray scales, the darker the colder). The thermal pattern includes footprints of turbulent wedges generated by surface roughness, mostly originating close to the leading edge and merging into the natural laminar-turbulent transition (hereafter referred to as the transition). In this case, turbulent regions appear colder since the rotor blade is warmed up by the morning sun while the air is still cold from the night.

Certainly not every turbulent wedge corresponds to a severe defect, but Fig. 1 draws a vivid picture of the condition of new rotor blades. Typical surface faults at a young age of a rotor blade are residue from manufacturing, damages from transportation and first erosion pits. Since surface defects are in general to be avoided for economic and safety reasons, this study aims at proving the concept of IRT as a remotely operating inspection method that is capable of providing information about the type of surface defect just from the flow formation mapped by the thermogram. This implies, for the first time, the following objectives:

- documented application of IRT as a surface inspection method for rotor blades of operating wind turbines, 
- detailed comparison of measured flow topology against resultant thermal footprint in aerodynamics.

In order to achieve these objectives, an argument is made consisting of three main steps. Step 1 covers the flow topology in the wake of generic, protruding surface defects using a simplified setup of a flat plate that is manipulated by different hemispherical turbulators. For each turbulator, the corresponding thermal footprint is determined, which allows for distinguishing the size of the turbulator by means of the thermogram only. In step 2, the formation of turbulent wedges induced by generic surface defects on an airfoil designed specifically for wind energy applications is investigated. This provides deeper insight into thermal features encountered in real wind turbines. Since inferior aerodynamic performance directly implies reduction of energy yield of a wind turbine, the impact of potential surface defects on the performance of the airfoil is then assessed by means of lift measurements. In step 3, the so far geometrically reduced consideration is extended to a three-dimensional rotor blade of a wind turbine employing a computational fluid dynamics (CFD) simulation. This investigation allows us to confirm that the flow patterns captured experimentally behind surface defects on static blade profiles also exist on rotating wind turbine blades. The simulations also provide insight into the three-dimensional behavior of the flow and contribute to clarify if IRT has the potential to capture surface damages on wind turbine blades that would otherwise remain "hidden" and undetectable by the power output signal.

Following these three steps within each section, the potential of IRT as a tool for remote surface damage detection in rotor blades of operating wind turbines on site is evaluated. After treating the used methods and experimental setups in Sect. 2, results are depicted and discussed in Sect. 3 with some concluding remarks in Sect. 4.

\section{Methods}

\subsection{Wind tunnel measurements of turbulent wedges on a flat plate}

First, the fundamental link between turbulators, the resultant flow topology and corresponding thermal appearance is investigated. Turbulent wedges induced by hemispherical turbulators are investigated in a simplified setup on a flat plate, as schematically shown in Fig. 2. BL transition is an unsteady process that emerges stochastically over a certain streamwise range on rotor blades. In order to capture this complex and delicate flow topology without interfering, it is essential to use non-intrusive techniques featuring high spatial resolution. A combination of stereoscopic particle image velocimetry (SPIV) and IRT is thus used here.

The SPIV system consists of two Phantom Miro M320S high-speed cameras and a Litron LDY300 laser. This enables SPIV measurements with a recording frequency of

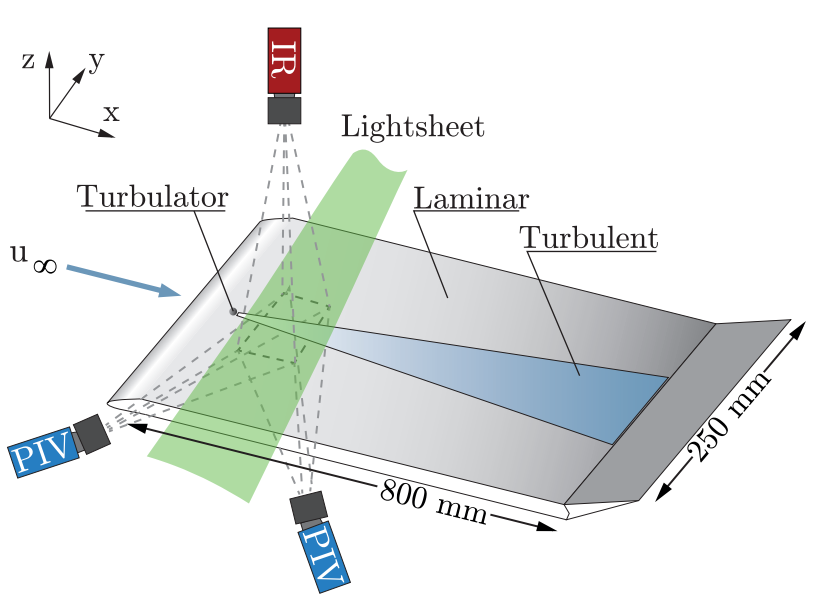

Figure 2. Experimental setup for SPIV and IRT measurements of a turbulent wedge emerging on a flat plate downstream of a turbulator.

695 velocity fields per second at a full resolution of $1920 \times$ $1200 \mathrm{px}^{2}$. The light sheet is adjusted parallel to the surface at a distance, $z=\delta_{u / 2}<1 \mathrm{~mm}$, where $50 \%$ of the free-stream velocity, $u_{\infty}$, is present in the laminar BL. Due to moderate growth of the BL, this height remains approximately constant over the region of interest. In this way, a sufficient accuracy (stereo residue $<0.5 \mathrm{px}$ ) and difference in velocity between the laminar BL and the wake of the turbulator is obtained.

Thermal measurements are carried out using an IRCAM Equus $327 \mathrm{k} \mathrm{M}$ infrared camera featuring a resolution of $640 \times 512 \mathrm{px}^{2}$. Since presented results are steady-state only, an integration time of $2 \mathrm{~ms}$ along with a thermal resolution of $\Delta T=15 \mathrm{mK}$ yields low-noise and detailed thermograms. Using a temperature difference $\delta T$ between surface and flow that enables mapping flow features onto the surface is referred to as the "method of temperature steps" (Tropea et al., 2007). Accordingly, this experimental setup exploits a $\delta T$ emerging from the wind tunnel warmed up by its fan and the cooler laboratory without any additional heating or cooling applied on the flat plate. Thermal contrast of turbulent regions remains recognizable as long as the heat capacity of the PVC plate and its internal equalization by thermal conduction are sufficient. The low thermal conductivity of PVC thus enables detailed mapping of flow features.

The infrared camera is adjusted perpendicular to the flat plate. Both measurement techniques, SPIV and IRT, cover congruent fields of view extending approximately $\Delta x \times$ $\Delta y=20 \times 70 \mathrm{~mm}^{2}$ in the wake of the turbulator ( $x$ and $y$ are denoted according to Fig. 2). A hemispherical turbulator with a diameter of $d=3.5 \mathrm{~mm}$ serves as a generic defect located at a distance of $\Delta x=100 \mathrm{~mm}$ from the leading edge of the flat plate. The black PVC plate (size: $800 \times 250 \mathrm{~mm}^{2}$ ) is provided with a high-precision hyperelliptic leading edge keeping the BL attached and laminar (Narasimha and Prasad, 1994). Obviously, the induced transition is strongly depen- 
dent on the Reynolds number and free-stream properties. Minimizing the impact of the latter, the experiments are performed in a wind tunnel (inlet: $250 \times 250 \mathrm{~mm}^{2}$, length: $2000 \mathrm{~mm}$, closed test section equipped with IRT window) featuring a low turbulence intensity of $T i<0.4 \%$. An increasing Reynolds number with respect to the height of the turbulator $-R e_{\mathrm{h}}=u_{\infty} d / v$, where $u_{\infty}$ denotes the freestream velocity and $v$ the kinematic viscosity - corresponds to a stronger destabilization of the BL and represents a more relevant surface defect. The impact of the turbulator is thus varied by changing $u_{\infty} \in[7 ; 17] \mathrm{m} \mathrm{s}^{-1}$, i.e., $R e_{\mathrm{h}} \in$ $[700 ; 1750]$.

\subsection{Wind tunnel measurements of turbulent wedges on airfoils}

The experimental setup shown in Fig. 3 allows us to compare aerodynamic forces with IRT measurements. In this way, the aerodynamic impact of turbulent wedges is investigated on a DU 91-W2-250 airfoil. The airfoil has dimensions of $S \times C=805 \times 300 \mathrm{~mm}^{2}$ where $S$ denotes the span and $C$ the chord length of the airfoil. It is mounted at the quarter chord point on an axis which is precisely turned by a stepper motor for setting the angle of attack (AoA). For accurate aerodynamic measurements, the high-precision airfoil is made of aluminum. In general, aluminum is not considered to be suitable for IRT applications because of high thermal conductivity blurring any thermal contrast by internal heat equalization (Tropea et al., 2007). To overcome this problem, the airfoil is covered with a matte black PVC foil (thickness $<100 \mu \mathrm{m}$ ) which inhibits the thermal conduction transversally to the surface while the geometry is negligibly changed (Reyer et al., 2006; Joseph et al., 2016). Such a configuration provides sharp thermal imaging with sufficient heat capacity to maintain temperature differences that emerge due to flow characteristics. Additionally, reflections in the infrared spectral range are minimized along with a maximized emissivity being crucial for reliable IRT.

Two experiments are performed with this airfoil. The first experiment focuses on qualitatively imaging turbulent wedges for direct comparison with results of Section 2.1. The wind tunnel is thus operated in a half-open setup without walls at the pressure and suction side of the airfoil providing an unobstructed view by IRT. The flow quality given by a turbulence intensity $T i<0.3 \%$ is yet preserved in the center of the test section allowing investigations on transition phenomena (Stabe and Langner, 1997). Inflow velocities of $u_{\infty} \in[0 ; 30] \mathrm{m} \mathrm{s}^{-1}$ result in Reynolds numbers up to $R e_{C}=600000$ with respect to the chord length.

For thermal imaging, an IRCAM Geminis 327k ML pro is used, providing a spatial resolution of $640 \times 512 \mathrm{px}^{2}$ and a thermal resolution of $\Delta T=15 \mathrm{mK}$. In comparison with Sect. 2.1, similar imaging quality is obtained by only using the detector for mid-wavelength infrared. To image turbulent wedges on the curved surface with minor optical distortion,

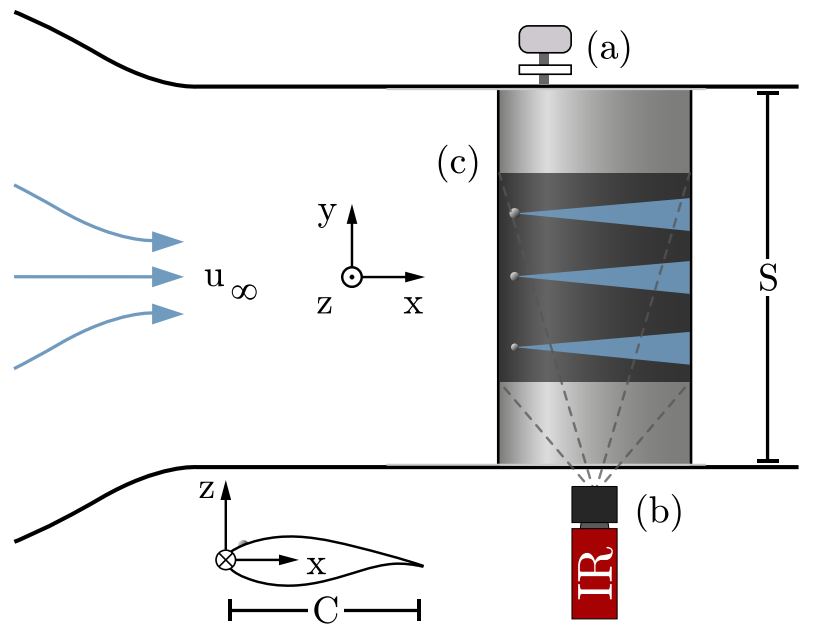

Figure 3. Experimental setup for lift (a) and IRT measurements (b) of a DU 91-W2-250 airfoil (c) modified with turbulators.

the infrared camera is adjusted perpendicular to the plane defined by the chord length of the airfoil and its span at zero AoA. The method of temperature steps is again applied using temperature differences between the up to $2^{\circ} \mathrm{C}$ cooled wind tunnel flow and the ambient air. The temperature difference is achieved by cooling the wind tunnel to about $2{ }^{\circ} \mathrm{C}$ below the ambient temperature of the air in the laboratory.

As defects on rotor blades usually occur on the suction side in distances $x$ from the leading edge of $x / C<0.13$ (Sareen et al., 2014), three hemispheric turbulators (see Fig. 3) of different diameters $d=1,2,3 \mathrm{~mm}$ are placed on the suction side at the chordwise position $x / C \approx 0.1$. In terms of the turbulator Reynolds number, $R e_{\mathrm{h}}$, the different sizes of the turbulators along with a variation in inflow velocity simulate both growing surface defects as well as spanwise position on a rotor blade (from root to tip).

A second version of this setup is used to investigate the impact of protruding surface defects on the performance of an airfoil at different AoA. The test section is closed to avoid interferences between the flow about the airfoil and the laboratory. To emphasize the aerodynamic impact and avoid interaction of turbulent wedges, five identical hemispherical turbulators $(d=2 \mathrm{~mm})$ are equidistantly positioned $(\Delta y / C=$ 0.5 ) on the airfoil at $x / C \approx 0.01$. Measurements are performed at $\operatorname{Re}_{C}=1000000$ without PVC foil. A Reynolds number on the order of $10^{6}$ is relevant to wind energy applications. The lift force is acquired by two load cells connected to the airfoil's rotary axis and decoupled from the possibly vibrating wind tunnel. Lift forces, $F_{\mathrm{L}}$, can be measured with an accuracy of $\Delta F / F_{\max }<1 \%$ at a temporal resolution of $f_{\mathrm{s}}=1 \mathrm{kHz}$, where $F_{\max }$ refers to the maximum load, $F_{\max }=500 \mathrm{~N}$, and $f_{\mathrm{s}}$ to the sampling frequency. 


\subsection{CFD simulation of turbulent wedges on rotor blades}

The experimental analyses are performed on static quasitwo-dimensional geometries. In order to estimate if the observed flow patterns behind turbulators can also be found in rotating, three-dimensional blades, CFD simulations of a wind turbine rotor with generic defects are performed. The baseline model is a two-bladed wind turbine with clean blades that has been simulated and validated against experimental results in Herráez et al. (2016). The turbine has a rotor diameter of $2 \mathrm{~m}$ and it employs the DU 96-W-180 airfoil along the whole blade span except at the cylindrical root connection to the hub. In full-scale rotor blades of wind turbines, this type of profile is typically used in the outboard region of the blade where most of the torque is generated and high local inflow velocities often lead to surface defects. Only rated operating conditions are considered in this work, i.e., axisymmetric inflow with a wind speed of $u_{\infty}=6 \mathrm{~m} \mathrm{~s}^{-1}$ and a rotational speed of $40 \mathrm{rads}^{-1}$, giving a tip speed ratio $\lambda \approx 7$. The Reynolds number with respect to the local chord length ranges between $R e_{C} \approx 1.4 \times 10^{5}$ at the root and $R e_{C} \approx 2.8 \times 10^{5}$ at the tip.

The simulations are performed with the open-source, finite-volume-based numerical toolbox OpenFOAM (2015). The focus of this work is on stationary effects caused by blade surface defects. Therefore, the computations are based on the Reynolds-averaged Navier-Stokes (RANS) method. For the convective terms, a linear upwind second-order discretization scheme is used. The viscous terms are discretized with a second-order central-differences scheme. The Coriolis and centrifugal forces are added to the RANS equations in order to account for the rotation of the system. The pressurevelocity coupling is enforced with the SIMPLE algorithm. Turbulence is modeled by means of the two-equation $\mathrm{k}-\omega$ model of Menter (1993).

The use of periodic boundary conditions allows us to exploit the symmetry of the rotor by simulating only one half of it. For the outer boundary of the domain, a hybrid DirichletNeumann boundary condition is used, which behaves like a Dirichlet condition in regions where the flow enters the domain (it enforces a predefined wind speed), and as Neumann condition in regions where the flow goes out (it predefines zero gradient). No-slip boundary conditions are applied to the blade walls. A so-called arbitrary mesh interface connects the inner and outer parts of the domain, which consists of two independent block-structured meshes. This allows us to independently control the mesh resolution in both regions, which in turn makes if possible to reduce the number of cells in regions where a fine mesh is not required (see also Herráez et al., 2016).

The computational mesh, which is created with the software Pointwise (Pointwise Inc, 2015), is of the blockstructured type; see Figs. 4 and 5. The domain is semispherical and the outer radius has a length $22 R$, where $R$ corresponds to the blade radius. The complete mesh contains

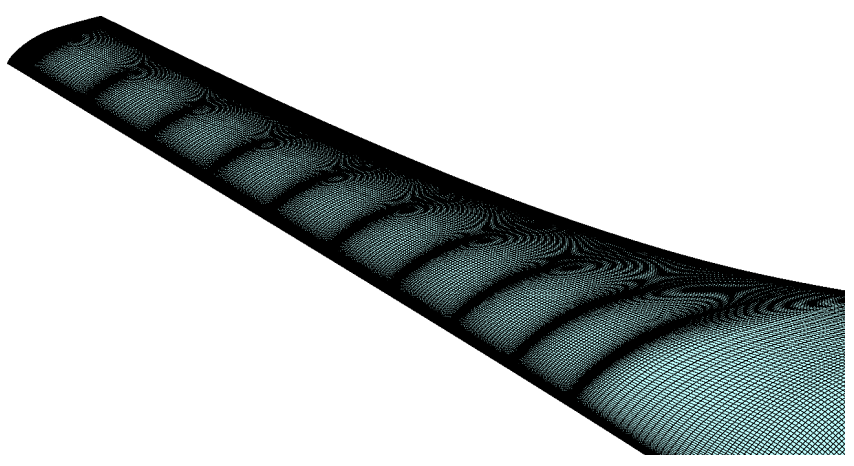

Figure 4. Surface mesh of the blade including 10 generic surface defects uniformly distributed in the range $0.5 R \leq r \leq 0.99 R$. The regions in the vicinity of turbulators present an increased cell density.

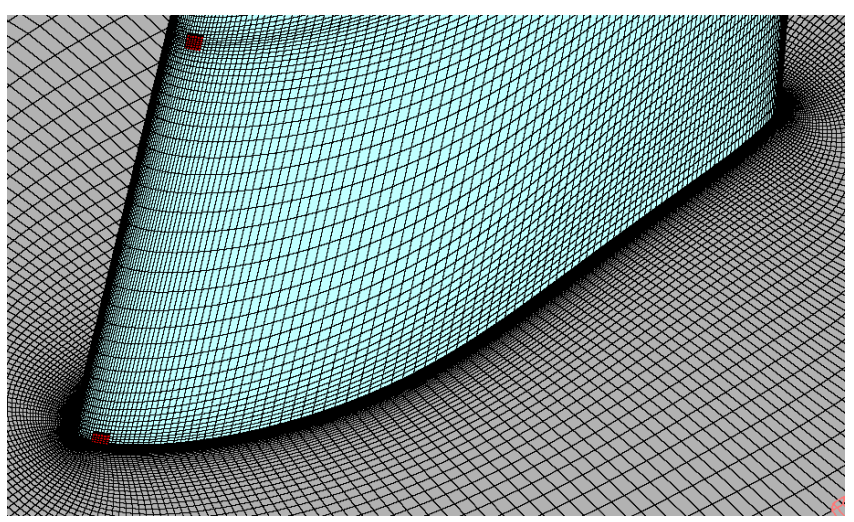

Figure 5. Surface mesh of the blade and volume mesh around it. Two generic surface defects are highlighted in red color.

$16.8 \times 10^{6}$ cells. The increased number of cells with respect to the mesh presented in Herráez et al. (2016) comes from the finer blade resolution in the spanwise direction, containing 507 cells along the span. This high spanwise resolution allows the blade surface cells in the proximity of generic surface defects to have an aspect ratio of approximately 1 . In the chordwise direction the resolution is 130 cells both in the pressure and suction sides of the blades. Ten turbulators are uniformly distributed in the spanwise range from $r=0.50 R$ to $r=0.95 R$ to significantly affect the BL flow while avoiding strong mutual interference of turbulent wedges. The distance between turbulators and the leading edge is $1 \%$ of the chord length, in analogy to the experimental setup presented in Sect. 2.2. The shape of the turbulators is chosen to be cuboid in order to reduce the complexity of the meshing process. The length, width and height of each turbulator is approx. $2 \times 2 \times 0.5 \mathrm{~mm}^{3}$, respectively. It is worth remarking that great care is put into having a high grid resolution in the proximity of turbulators and their wake in order to properly resolve the strong gradients taking place in that region. 
The height of the first cell in the normal direction to the blade surface is set to $5 \times 10^{-6} \mathrm{~m}$ in order to ensure that the $Y+$ value remains below 1 along the whole span for fully resolving the BL. The extraction of the AoA from the numerical results is accomplished by using the method presented in Herráez et al. (2018).

\section{Results and discussion}

\subsection{Characteristics of turbulent wedges on a flat plate}

The PVC plate material allows for detailed thermograms that map the flow topology within turbulent wedges. According to the given thermal response time, the temporal mean over 1800 instantaneous velocity fields $(\approx 2.6 \mathrm{~s})$ obtained by SPIV is recorded in the wake of different turbulators. In Fig. $6 \mathrm{a}-\mathrm{c}$, the velocity magnitude normalized by $u_{\infty}$ is color coded and visualized for three characteristic Reynolds numbers, $R e_{\mathrm{h}} \in$ [700; 1750] (cf. Traphan et al., 2015). The position in $x$ and $y$ directions refer to the location of the turbulator defining the origin at the position $(x, y)=(0,0)$.

Since these measurements are taken at a distance from the flat plate where the undisturbed BL reaches $\langle u\rangle / u_{\infty}=0.5$ (cf. Sect. 2.1), $\langle u\rangle / u_{\infty} \neq 0.5$ implies regions of higher or lower mean velocity that can be associated with an underlying mean vortex structure. The flow topology around a tall obstacle is known to present vortex shedding from its top, along with horseshoe vortices winding around it, separating and propagating downstream (Euler and Herget, 2012). In a measuring plane parallel to the surface, a quasi-steady vortex within the BL propagating downstream appears as a region of higher mean velocity where the flow is moving towards the surface and a region of lower velocity where the flow is coming from the surface. The mean velocity field obtained from SPIV is shown in Fig. 6a for $R e_{\mathrm{h}}=700$. There are four horizontal lines displaying evidence of two counterrotating horseshoe vortices (each consisting of one pair of velocity deficit and overshoot). These vortices are localized without spreading transversely. In terms of protruding surface defects, a minor defect forms a turbulent wedge which is not fully developed in its wake.

Increasing the Reynolds number towards $R e_{\mathrm{h}}=1150$ reveals a characteristic change in the shape of wedges (see Fig. 6b). Perturbations arising from the turbulator start to spread symmetrically around $y=0 \mathrm{~mm}$. The BL becomes more perceptive for perturbations forming a ragged contoured wedge in the mean velocity field. The two trailed vortices from the turbulator induce another counter-rotating vortex pair downstream of $x \approx 52 \mathrm{~mm}$. This cascade continues while propagating downstream. When the Reynolds number is increased towards $R e_{\mathrm{h}}=1750$ (see Fig. 6c), perturbations continuously spread and form a smoothly shaped turbulent wedge in the mean velocity field.

One initial conclusion regarding the SPIV investigation of a flat plate is that basically three flow regimes can be identi- fied for different Reynolds numbers. Following the flow stability, these regimes are termed subcritical, critical and supercritical. Respective Reynolds numbers are denoted as $R e_{\mathrm{h}}^{\mathrm{sub}}$, $R e_{\mathrm{h}}^{\text {crit }}$ and $R e_{\mathrm{h}}^{\text {sup }}$. The significance of a surface defect can thus be evaluated by means of the velocity field. However, performing SPIV measurements on operating rotor blades of wind turbines is virtually impossible for remote maintenance. In the next step, the thermal patterns of subcritical, critical and supercritical turbulent wedges are captured by IRT and related to present flow features. This determines the capability of IRT distinguishing surface defects by means of the thermogram only.

A thermogram for $R e_{\mathrm{h}}=R e_{\mathrm{h}}^{\text {crit }}$ consisting of two laterally shifted and stitched thermograms is presented in the background of Fig. 6d. It is overlaid by an extract of the corresponding velocity field obtained by SPIV whose color coding is the same as in Fig. 6b. The thermogram is color coded in raw digital values to match the color range of the velocity field allowing easier comparison. The dynamic range of the thermogram is roughly $2^{\circ} \mathrm{C}$.

Both measurement results show good qualitative agreement. Each flow feature in the velocity map has a visual counterpart in the thermogram. This finding holds true for the other Reynolds numbers, $R e_{\mathrm{h}}^{\text {sub }}$ and $R e_{\mathrm{h}}^{\text {sup }}$ (not shown here). As a conclusion for a flat plate, IRT is capable of appropriately representing (quasi-)stationary flow features in the BL with moderate experimental effort. In the wake of a turbulator, flow features depend on details of their generation providing evidence for the significance of a turbulator and surface defect by means of the thermogram only. In order to check whether surface material or curvature affects the so-far uncovered relations, turbulent wedges emerging on an aluminum airfoil are studied in the following Sect. 3.2. Besides, the aerodynamic impact of turbulators applied to this airfoil is assessed.

\subsection{Impact of turbulent wedges on airfoils}

Different stages of surface defects on a DU 91-W2-250 profile are simulated by a constant free-stream velocity with varying turbulator heights, $d / 2$, yielding different Reynolds numbers $R e_{\mathrm{h}}$, in particular $R e_{\mathrm{h}}^{\text {sub }}, R e_{\mathrm{h}}^{\text {crit }}$ and $R e_{\mathrm{h}}^{\text {sup }}$. The resultant thermogram of three turbulent wedges generated by hemispherical turbulators $d=1,2,3 \mathrm{~mm}$ (from bottom to top) is presented for $R e_{C}=3 \cdot 10^{5}$ in Fig. 7a. Please note that $\operatorname{Re}_{C}$ refers to the chord length and thus reflects the global flow about the airfoil. The PVC foil yields a distinct contrast with detailed thermal features. The laminar-turbulent transition at $x / C \approx 0.5$ as well as the three turbulent wedges is clearly visible. The smallest turbulator at the bottom induces a confined wake in its near-field forming a nearly straight line. Compared with Fig. 6a, as well as in terms of $R e_{\mathrm{h}}$, this turbulent wedge is initially subcritical. However, the thermogram shows only one straight line instead of four. Given that the pair of counter-rotating horseshoe vortices identified on 


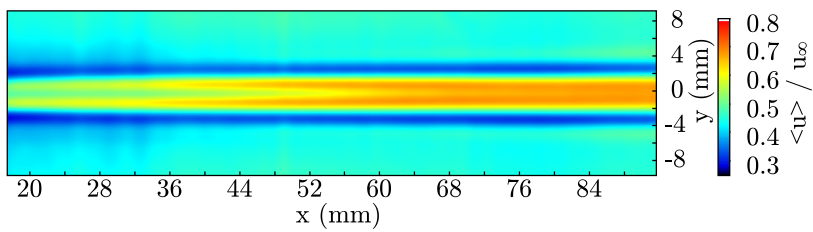

(a) $R e_{\mathrm{h}}=700$.

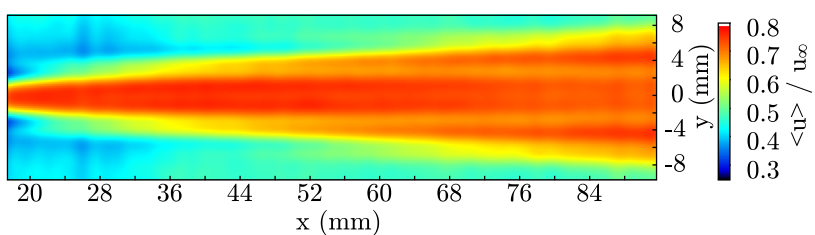

(c) $R e_{\mathrm{h}}=1750$.

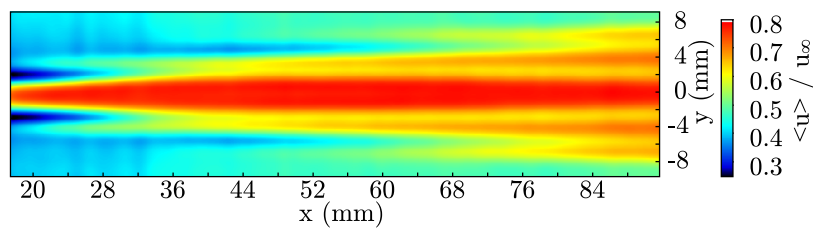

(b) $R e_{\mathrm{h}}=1150$.

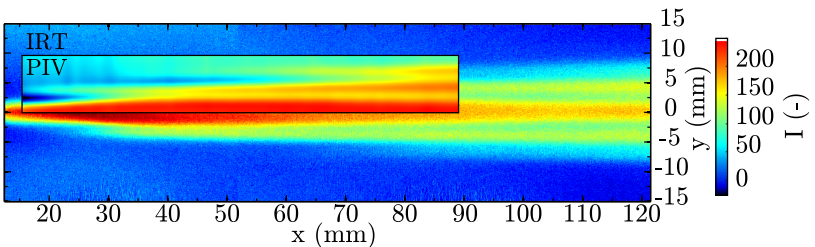

(d) $R e_{\mathrm{h}}=1150$.

Figure 6. Averaged SPIV measurement of a turbulent wedge on a flat plate downstream of a generic surface defect at different Reynolds numbers in (a)-(c). Overlaying the thermogram with SPIV results in (d), which allows direct comparison with each other. Color coding of the thermogram represents digital values, $I$, spanning roughly $2{ }^{\circ} \mathrm{C}$. Limits are chosen to match the dynamic range of the SPIV measurement. Defect $d$ at $(x, y)=(0,0)$; velocity magnitude $u$ normalized by free-stream velocity $u_{\infty}$; light sheet parallel to surface.

a flat plate (cf. Fig. 6a) are also present in this subcritical wedge, the thermogram suffers from limited spatial resolution at expense of imaging the major part of the airfoil. However, the subcritical turbulent wedge can still be clearly identified on the aluminum airfoil.

While traveling over the airfoil, the receptivity of the BL for perturbations increases. Initially subcritical, the turbulent wedge thus evolves to a critical one. At $x / C \approx 0.25$, the wake of the small turbulator, $d=1 \mathrm{~mm}$, destabilizes and spreads out. Even without resolving the characteristic raggedly contoured wedge in details (cf. Fig. 6b) the critical behavior is identified. Considering the thermal footprint in the wake of major turbulators in the middle and top of Fig. 7a, two supercritical turbulent wedges are formed. Following arguments of Sect. 3.1, perturbations continuously spread out and form a smoothly shaped turbulent wedge. Nevertheless, both supercritical wedges reveal differences in appearance at respective origin and regarding transversal propagation. Beyond the three categories of subcritical, critical and supercritical wedges, such characteristics enable further distinction between significant surface defects. This is confirmed by looking at the thermal signature for a higher Reynolds number, $R e_{C}=600000$, in Fig. 7b. In this case, all turbulent wedges are supercritical. Compared with $\operatorname{Re}_{C}=300000$, horseshoe vortices induce the turbulent wedge closer to the turbulator while the wedge broadens with increasing $R e_{\mathrm{h}}$. In this way, a variety of indicators for assessing the relevance of a surface defect are provided.

In the sense of a feasibility analysis, IRT is shown to be able to capture sufficient characteristics for distinguishing different stages of turbulent wedges generated by generic surface defects on a flat plate as well as on airfoils. However, the question remains whether there is a need for detecting such surface defects at all. The aerodynamic impact is thus quantified by lift measurements according to the experimental setup described in Sect. 2.2.

Wind turbine blades usually operate at an effective local AoA near the maximum lift-to-drag ratio, $c_{\mathrm{L}} / c_{\mathrm{D}}$, which is typically $\alpha \approx 7-9^{\circ}$ (Timmer and Van Rooij, 2003). Characteristics of the atmospheric BL, e.g., gusts, wind shear or wakes of adjacent obstacles, modulate the mean AoA towards fluctuations easily exceeding the stall angle of the airfoil. For this reason, the analysis of aerodynamic performance is focused on an angular range of $\alpha \in[0 ; 20]^{\circ}$. The mean lift coefficient, $c_{\mathrm{L}}$, as a function of AoA including corresponding fluctuations in terms of standard deviation is depicted in Fig. 8. The airfoil with turbulators applied is denoted as "modified" and without as "clean".

The modified airfoil performs consistently worse than the clean one. The deficit in lift increases towards the AoA of maximum lift, $\alpha_{\max }=10^{\circ}$, and decreases beyond. Since lift correlates to some degree with energy output, a lift deficit of up to $5 \%$ at $\alpha_{\max }$ is generally unfavorable for a wind turbine. Inferior performance of the modified airfoil is also reflected by a lift-to-drag ratio, $c_{\mathrm{L}} / c_{\mathrm{D}}$, which is almost $10 \%$ lower, as seen in the insert of Fig. 8. In order to examine the lift stability that strongly affects a wind turbine in terms of fatigue, we take a closer look at the maximum lift range. This reveals a striking difference between the two conditions. In the post-stall range, particularly at $\alpha=12^{\circ}$, the modified airfoil exhibits fluctuations of lift and pitching moment increased by more than $\Delta \sigma\left(c_{\mathrm{L}}\right) \approx 50 \%$ and $\Delta \sigma\left(c_{\mathrm{M}}\right) \approx 80 \%$, respectively. Under real inflow conditions inducing off-design AoA, the presence of protruding surface defects on airfoils potentially induces more flow instabilities and, in turn, larger dynamic loads leading to premature material fatigue. 


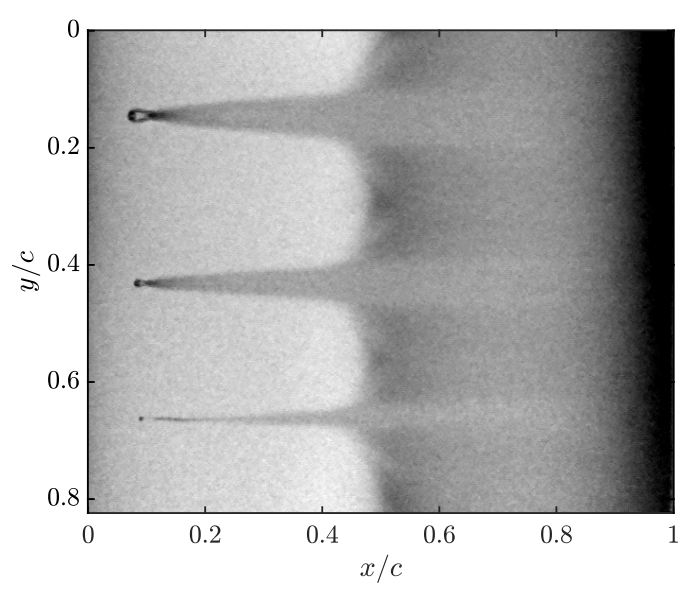

(a) $\operatorname{Re} \mathrm{C}=3 \cdot 10^{5}$.

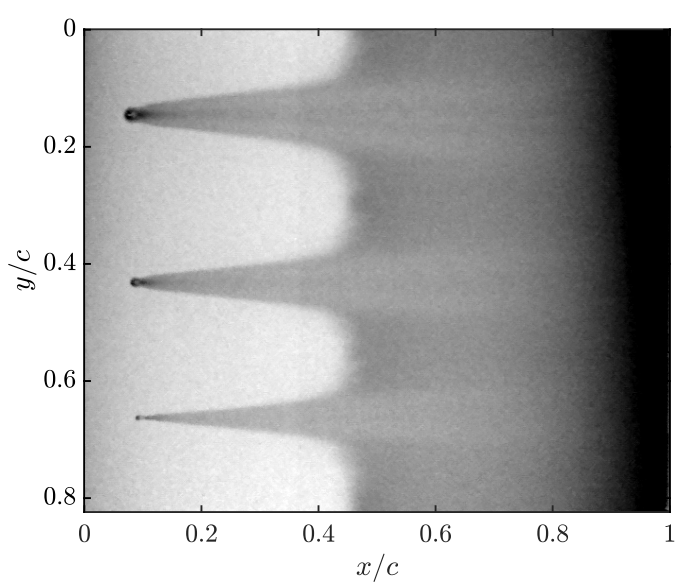

(b) $R e_{\mathrm{C}}=6 \cdot 10^{5}$.

Figure 7. Thermogram of turbulent wedges generated by three hemispherical turbulators of diameter $d=1,2,3 \mathrm{~mm}$ (from bottom to top) on a DU 91-W2-250 profile $\left(\alpha=12^{\circ}\right)$ located at $x / C=0.09$ and displaced by $\Delta y / C=0.5$ for two different Reynolds numbers. Depiction in gray scales and color coded in digital values $\left(\Delta T<2{ }^{\circ} \mathrm{C}\right)$.

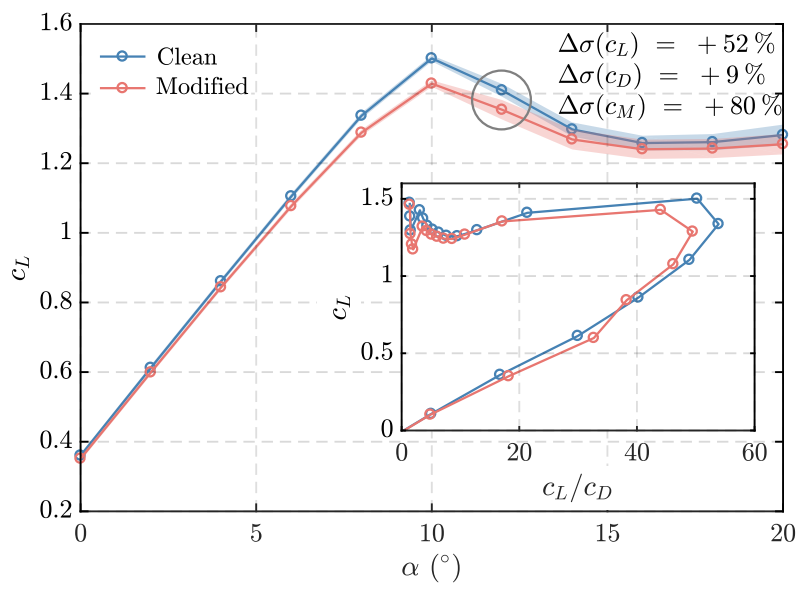

Figure 8. Lift coefficient $c_{\mathrm{L}}$ over angle of attack (AoA) of a clean DU 91-W2-250 profile (- - ) and a modified one with generic surface defects $(--)$. The insert shows measured lift coefficients and corresponding lift-drag ratios. The respective $\Delta \sigma$ specifies the increase in fluctuations of the associated quantity in terms of standard deviation.

\subsection{Turbulent wedges on rotor blades}

The experimental results have shown that IRT provides valuable information for detecting protruding surface defects on quasi-two-dimensional airfoils. However, it still remains open if similar flow patterns in the wake of turbulators can be found on three-dimensional, rotating wind turbine blades. This is indeed a crucial point for estimating the potential of IRT for wind turbine inspection. In order to address this issue, a numerical simulation of an operating wind turbine with turbulators is performed and presented in the following. Furthermore, the analysis of numerical results provides detailed flow information that helps to verify our interpretations from the IRT experiments.

As stated in Sect. 2.3, the baseline wind turbine numerical model used in this work is validated against SPIV measurements in Herráez et al. (2016). Therefore, this section focuses only on the aerodynamic behavior of the modified blade surface with generic surface defects. The flow patterns obtained from the wall shear stress computed by the simulations are qualitatively compared with the experimental results of Sects. 3.1 and 3.2.

Figure 9 displays iso-surfaces of the vorticity in the chordwise direction, $\omega_{x}$, for the six outer-most turbulators. It is observed that the flow downstream of the turbulators is characterized by well defined vortical structures. From the figure, it is also clear that the wake of each turbulator is characterized by two counter-rotating vortices: the vortex just outboard of the turbulator rotates in the same direction as the tip vortex (also depicted in the figure), whereas the vortex just inboard of the turbulator rotates in the opposite direction. These vortices give rise to the characteristic turbulent wedge in the BL described in Sect. 3.1 and 2.2.

Figure 10 provides further insight into the emergence of the turbulent wedges. In the figure, the flow around a single turbulator is observed from the leading edge by means of limiting streamlines representing the wall-bounded flow. In order to illustrate the thermal footprint of the flow, the so-called Reynolds analogy is applied (Kakac and Yener, 1994). According to that, the magnitude of the wall shear stress, $\tau_{\mathrm{w}}$, around a generic defect images the thermal map that an infrared camera would capture. The footprint of vortical structures winding around the turbulator is clearly visible. The resulting horseshoe vortices impinge onto the blade surface behind the turbulator and then propagate downstream, as also seen in Fig. 9. These results verify the interpretations 


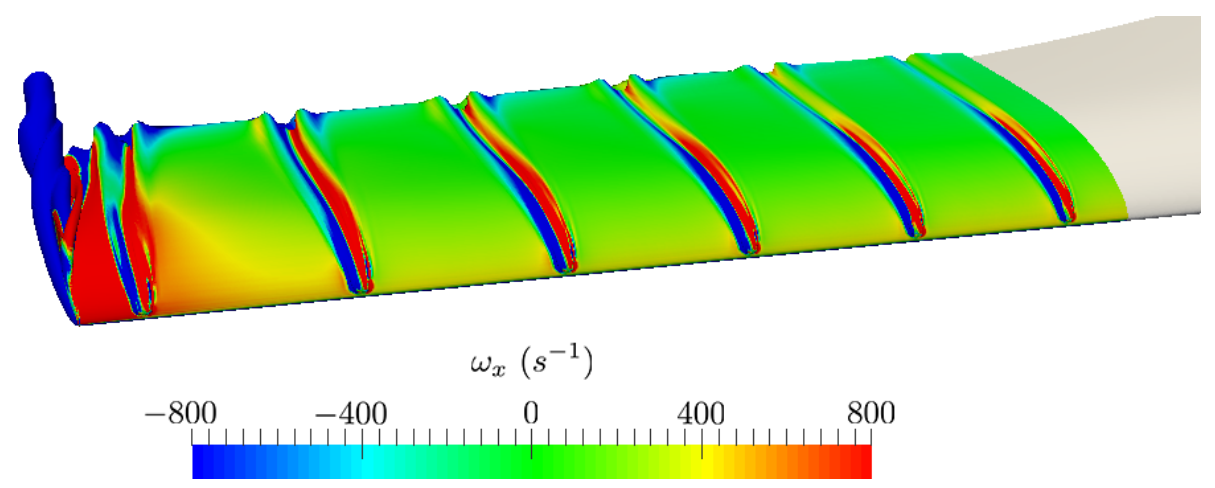

Figure 9. Iso-surface of the vorticity in the chordwise direction past six turbulent wedges on the suction side of a rotating blade.

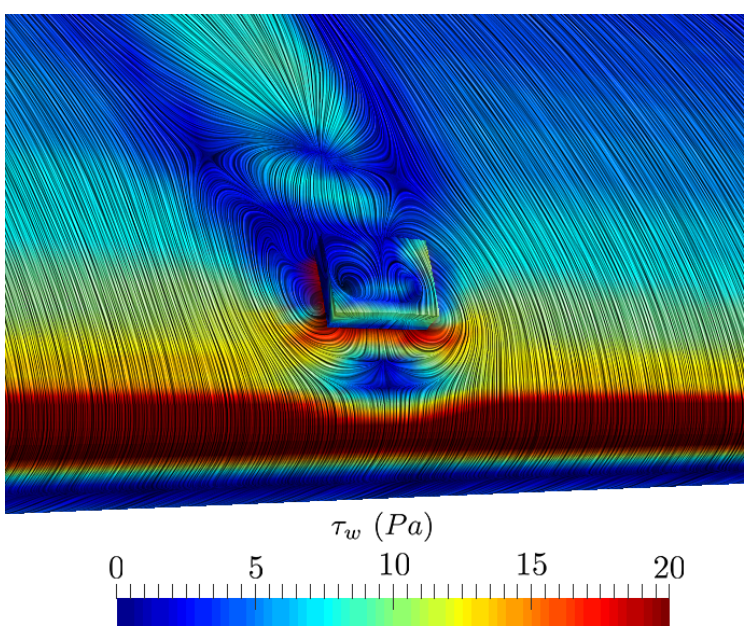

Figure 10. Detail of the limiting streamlines over a turbulator located at $r / R=0.66$ as observed from the leading edge. The magnitude of the wall shear stress, $\tau_{\mathrm{W}}$, is depicted over the blade surface. The footprint of the flow around the turbulator, which is located in the center of the figure, is characterized by a set of vortical structures winding around it.

of the experimental results discussed in Sects. 3.1 and 3.2 and demonstrate that the flow patterns around generic surface defects on rotating wind turbine blades do not present significant differences with respect to the static flat plate or blade section cases.

The magnitude of the wall shear stress on the suction side of one total rotor blade is shown in Fig. 11. The figure is depicted in gray scales with arbitrary units for easier comparison with Fig. 7. As it can be seen, the turbulent wedges are clearly visible for all radial positions. This is also true for the outermost location in spite of the strong influence of the tip vortex, which makes the turbulent wedge to be deflected towards the inner part of the blade. In general, these turbulent wedges highly resemble the ones obtained from the thermal footprints of the flat plate (cf. Fig. 2) and the airfoil (cf. Fig. 7).
However, the wakes of the turbulators in the inner region seem to vanish sooner in the blade than in the airfoil. This might be an artifact of the simulation attributed to the high dissipation of RANS models. For the other blade turbulators, a greater similarity with the IRT results is achieved. It is worth recalling that the turbulator-based Reynolds number $R e_{\mathrm{h}}$ increases linearly from the innermost turbulator $(r / R=$ $0.5)$ to the outermost one $(r / R=0.99)$ owing to the continuous increase in effective local inflow velocity from the root to the tip. From Table 1, it is clear that the four innermost turbulators are in the subcritical range introduced in Sect. 3.1. The fifth and sixth turbulators can be considered to be in the critical range. The four outermost ones belong to the supercritical category.

The turbulence model $\mathrm{k}-\omega$ shear stress transport (SST) assumes fully turbulent flow. This is the reason why the numerical results obtained from the blade simulation (Fig. 11) do not present a transition from laminar to turbulent flow, in opposition to the experimental results on the airfoil (Fig. 7). It is also worth recalling that different blade section types are employed for the blade and the experimentally tested airfoil (DU 96-W-180 and DU 91-W2-250, respectively). The angle of attack also differs between both applications: $\alpha=12^{\circ}$ for the airfoil and $\alpha \approx 7^{\circ}$ for all turbulator locations along the blade. This indicates that the flow patterns on the wake of turbulator-type surface defects are comparable for a wide range of conditions.

In Fig. 8, the performance of the DU 91-W2-250 airfoil is shown to be significantly reduced at AoA close to the stall onset when turbulators are applied to the suction side. However, in the wind turbine simulation no significant change in the aerodynamic performance is detected with respect to the baseline case. Several reasons exist for this behavior: on the one hand, the angle of attack for $r / R \geq 0.5$ is $\alpha \approx 7^{\circ}$ at all radial positions where the turbulators are located. This is a few degrees below the stall angle, so the influence of the turbulators is correspondingly expected to play a minor role (cf. Fig. 8). Furthermore, previous experiments have shown that the DU 96-W-180 airfoil (used in the simulated blade) presents low sensitivity to leading edge roughness (Timmer 


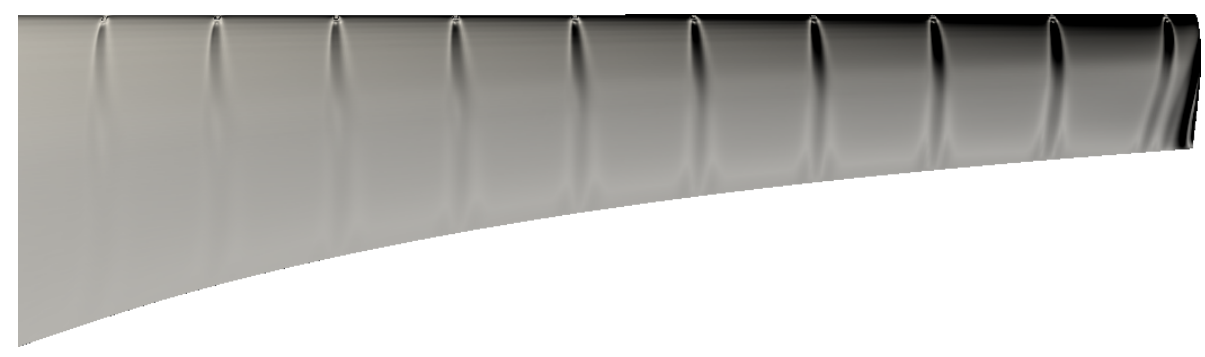

Figure 11. Distribution of wall shear stress obtained from the RANS simulation of a rotor blade with 10 turbulators equally distributed along the span in the range $0.5 R \leq r \leq 0.99 R$ and localized at the same relative chordwise position $x / C=0.01$. This figure resembles the representation resulting from a thermogram (Reynolds analogy; see Kakac and Yener, 1994). Gray scales in arbitrary units for facilitating the comparison with Fig. 7.

Table 1. Local turbulator-based Reynolds number $R e_{\mathrm{h}}$ and local chord-length-based Reynolds number $R e_{\mathrm{c}}$ as a function of the radial position $r / R$. The critical Reynolds number introduced in Sect. 3.1 is $R e_{\mathrm{h}} \approx 1000$, corresponding to the fifth and sixth turbulator (cf. Figs. 6 and 7 ).

\begin{tabular}{lrrrrrrrrrr}
\hline$r / R$ & 0.50 & 0.55 & 0.60 & 0.66 & 0.71 & 0.77 & 0.82 & 0.88 & 0.94 & 0.99 \\
$R e_{\mathrm{h}}$ & 698 & 768 & 837 & 921 & 991 & 1075 & 1145 & 1229 & 1312 & 1382 \\
$R e_{C}$ & $2.01 \times 10^{5}$ & $1.98 \times 10^{5}$ & $1.96 \times 10^{5}$ & $1.92 \times 10^{5}$ & $1.91 \times 10^{5}$ & $1.88 \times 10^{5}$ & $1.87 \times 10^{5}$ & $1.85 \times 10^{5}$ & $1.83 \times 10^{5}$ & $1.81 \times 10^{5}$
\end{tabular}

and Van Rooij, 2003). On the other hand, the sensitivity of the DU 91-W2-250 airfoil (used in the experimental test) to surface roughness is known to be substantially larger (Timmer and Van Rooij, 2003).

Figure 12 displays the distribution of the pressure coefficient, $c_{\mathrm{p}}$, along the chord for the radial position $r / R=0.94$, corresponding to the location of one turbulator. Additionally, the figure displays the corresponding $c_{\mathrm{p}}$ distributions $5 \mathrm{~mm}$ above and below the surface defect. As it can be seen, the surface defect only has a strong influence on the $c_{\mathrm{p}}$ distribution in the first $20 \%$ of the chord length. In that region, the surface defect leads to an increased suction peak and to a sudden pressure rise just after the suction peak, resulting in a local valley. Both effects counteract each other. Correspondingly, the impact of the studied surface defects on the power output is negligible. This indicates that IRT has the potential to detect surface defects long before they are noticeable in the power output signal.

\section{Concluding remarks}

The results of the present study qualify IRT as a monitoring method for rotor blades of operating wind turbines. It is shown that the presence of blade surface defects can be easily analyzed by means of thermograms.

The use of a RANS simulation of a wind turbine blade has shed light onto the underlying physical mechanisms governing the flow patterns behind generic surface defects. Furthermore, it allowed us to demonstrate that the flow phenomena observed in static quasi-two-dimensional profiles in the wind tunnel also apply to rotating three-dimensional wind turbine blades. The analysis of the numerical results also revealed that the use of IRT has the potential to detect surface defects

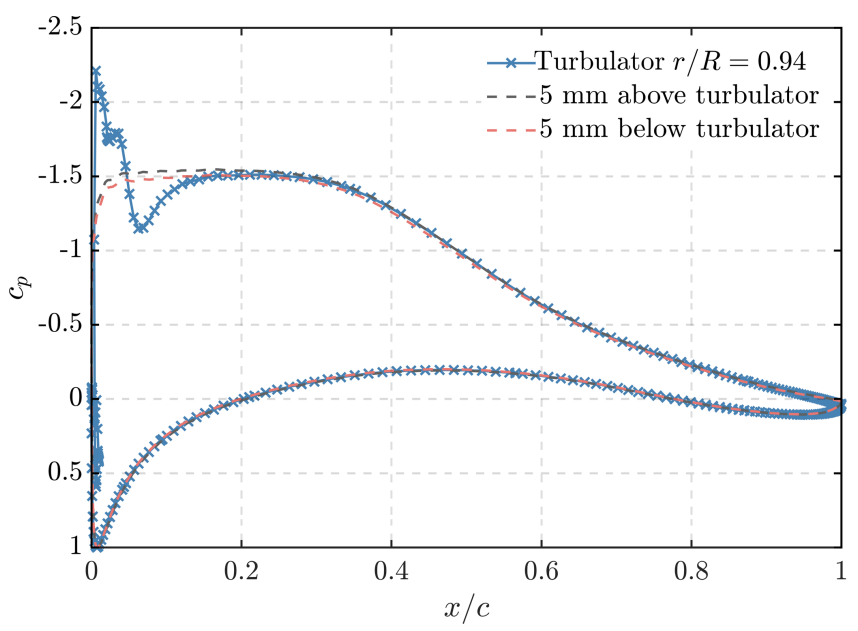

Figure 12. Distribution of the pressure coefficient $c_{\mathrm{p}}$ at the blade section $r / R=0.94$, corresponding to the location of one of the blade surface defects, as well as $c_{\mathrm{p}}$ distributions $5 \mathrm{~mm}$ above and $5 \mathrm{~mm}$ below the mentioned location.

that otherwise would remain "hidden" for the power output signal. Under off-design conditions in the near-stall range, as regularly encountered in the atmospheric BL, our aerodynamic measurements in the wind tunnel indicate that surface defects induce augmented dynamic loads.

As a next step, IRT needs to be used in practice on degraded full-scale rotor blades with complex damage patterns (preliminary on-site measurements gave already promising results). An extensive parameter study with a variety of differently shaped surface defects still needs to be performed. In particular, the extension from raised surface defects, as fundamentally treated in the present study, to pits, as char- 
acteristic of erosion, should be investigated. Additionally, a detailed analysis of the role of the Reynolds number on the flow patterns behind surface defects needs to be addressed in the future.

As this study shows, IRT is a handy, robust, non-intrusive and easily applicable experimental technique that enables us to image a variety of flow phenomena on aerodynamic subjects. We thus believe that IRT has the potential to allow for an unprecedented level of reliability and detail in monitoring the condition of large lifting surfaces under different operating conditions.

Data availability. Experimental data (SPIV, IRT, lift and drag) can be made available upon request.

Author contributions. DT performed the SPIV and IRT experiments and wrote most parts of the article. IH carried out the simulations and contributed to writing some parts of the manuscript. DT and IH also performed the analysis of the experimental and numerical results. PM and FS contributed to the IRT experimental campaign. JP and GG supervised all stages of the work.

Competing interests. The authors declare that they have no conflict of interest.

Acknowledgements. Gratitude goes to Otto Lutz, who shared his experience on defects of rotor blades as an authorized expert on fiber composite materials with us. Furthermore, this research has been supported by the Federal Ministry of Economic Affairs and Energy, Germany (BMWi, funding code FKZ 0325401A).

Edited by: Mingming Zhang

Reviewed by: Xiao Chen and one anonymous referee

\section{References}

Bak, C., Gaunaa, M., Olsen, A. S., and Kruse, E. K.: What is the critical height of leading edge roughness for aerodynamics?, J. Phys. Conf. Ser., 753, 022023, https://doi.org/10.1088/17426596/753/2/022023, 2016.

Carlomagno, G. M. and Cardone, G.: Infrared thermography for convective heat transfer measurements, Exp. Fluids, 49, 11871218,2010

Ciang, C. C., Lee, J.-R., and Bang, H.-J.: Structural health monitoring for a wind turbine system: a review of damage detection methods, Meas. Sci. Technol., 19, 122001, https://doi.org/10.1088/0957-0233/19/12/122001, 2008.

Corten, G. P. and Veldkamp, H. F.: Aerodynamics: Insects can halve wind-turbine power, Nature, 412, 41-42, 2001.

Dollinger, C., Balaresque, N., Sorg, M., and Fischer, A.: IR thermographic visualization of flow separation in applications with low thermal contrast, Infrared Phys. Techn., 88, 254-264, 2018 a.
Dollinger, C., Sorg, M., Balaresque, N., and Fischer, A.: Measurement uncertainty of IR thermographic flow visualization measurements for transition detection on wind turbines in operation, Exp. Therm. Fluid Sci., 97, 279-289, 2018b.

Ehrmann, R. S., White, E. B., Maniaci, D. C., Chow, R., Langel, C. M., and Dam, C. P. V.: Realistic Leading-Edge Roughness Effects on Airfoil Performance, in: 31st AIAA Applied Aerodynamics Conference, American Institute of Aeronautics and Astronautics, https://doi.org/10.2514/6.2013-2800, 2013.

Euler, T. and Herget, J.: Controls on local scour and deposition induced by obstacles in fluvial environments, Catena, 91, 35-46, 2012.

Ghoshal, A., Sundaresan, M. J., Schulz, M. J., and Pai, P. F.: Structural health monitoring techniques for wind turbine blades, J. Wind Eng. Ind. Aerod., 85, 309-324, 2000.

Herráez, I., Akay, B., van Bussel, G. J. W., Peinke, J., and Stoevesandt, B.: Detailed analysis of the blade root flow of a horizontal axis wind turbine, Wind Energ. Sci., 1, 89-100, https://doi.org/10.5194/wes-1-89-2016, 2016.

Herráez, I., Daniele, E., and Schepers, J. G.: Extraction of the wake induction and angle of attack on rotating wind turbine blades from PIV and CFD results, Wind Energ. Sci., 3, 1-9, https://doi.org/10.5194/wes-3-1-2018, 2018.

Horstmann, K., Quast, A., and Redeker, G.: Flight and wind-tunnel investigations on boundary-layer transition, J. Aircraft, 27, 146150, 1990.

Joseph, L. A., Borgoltz, A., and Devenport, W.: Infrared thermography for detection of laminar-turbulent transition in low-speed wind tunnel testing, Exp. Fluids, 57, 77 pp., https://doi.org/10.1007/s00348-016-2162-4, 2016.

Kakac, S. and Yener, Y.: Convective Heat Transfer, Second Edition, CRC Press, 2nd Edn., 1994.

Kucaba-Piętal, A., Stasicki, B., Politz, C., Roloff, C., Boden, F., Jentink, H., de Groot, K., Szumski, M., Valla, M., Póltora, P., Szczerba, P., James, S., Kirmse, T., and Weikert, T.: AIM ${ }^{2}$ Advanced Flight Testing Workshop: Handbook of advanced inflight Measurement Techniques, Books on Demand, 1 Edn., 2013.

Kuester, M. S. and White, E. B.: Structure of turbulent wedges created by isolated surface roughness, Exp. Fluids, 57, $47 \mathrm{pp}$. https://doi.org/10.1007/s00348-016-2140-x, 2016.

Li, D., Ho, S.-C. M., Song, G., Ren, L., and Li, H.: A review of damage detection methods for wind turbine blades, ADV Mater. Res.-Switz., 24, 033001, https://doi.org/10.1088/09641726/24/3/033001, 2015.

Liu, P., Sohn, H., and Park, B.: Baseline-free damage visualization using noncontact laser nonlinear ultrasonics and state space geometrical changes, ADV Mater. Res.-Switz., 24, 065036, https://doi.org/10.1088/0964-1726/24/6/065036, 2015.

Márquez, F. P. G., Tobias, A. M., Pérez, J. M. P., and Papaelias, M.: Condition monitoring of wind turbines: Techniques and methods, Renew. Energ., 46, 169-178, 2012.

Menter, F.: Zonal two equation $\mathrm{k}-\omega$ turbulence models for aerodynamic flows, AIAA J., 93, 2906, https://doi.org/10.2514/6.19932906, 1993.

Narasimha, R. and Prasad, S.: Leading edge shape for flat plate boundary layer studies, Exp. Fluids, 17, 358-360, 1994.

OpenFOAM: OpenFOAM: the open source CFD toolbox, available at: http://www.openfoam.com/, last access: 28 May 2015. 
Pointwise Inc: Pointwise Inc, version 17.3R2, available at: https: //www.pointwise.com/, last access: 28 May 2015.

Reyer, M., Rudolph, I., and Nitsche, W.: Investigations into the visualization and quantification of wall shear stress distributions using infrared thermography, in: Proc. of the 25th AIAA Aerodynamic Measurement Technology and Ground Testing Conference, AIAA-2006-3840, 2006.

Richter, K. and Schülein, E.: Boundary-layer transition measurements on hovering helicopter rotors by infrared thermography, Exp. Fluids, 55, 1-13, 2014.

Rogalski, A.: Recent progress in infrared detector technologies, Infrared Phys. Techn., 54, 136-154, 2011.

Rudolph, I., Reyer, M., and Nitsche, W.: Infrared-based visualization of wall shear stress distributions, Note. N. Fl. Mech. Mul. D., 106, 237-246, 2009.

Sareen, A., Sapre, C. A., and Selig, M. S.: Effects of leading edge erosion on wind turbine blade performance, Wind Energy, 17, 1531-1542, 2014.

Soltani, M. R., Birjandi, A. H., and Moorani, M. S.: Effect of surface contamination on the performance of a section of a wind turbine blade, Sci. Iran., 18, 349-357, 2011.

Stabe, U. and Langner, D.: Messung und Simulation systematischer Fehler des Ultraschallanemometers und deren Auswirkungen auf statistische Größen, Diploma thesis, University of Oldenburg, 1997.
Timmer, W. and Schaffarczyk, A.: The effect of roughness at high Reynolds numbers on the performance of aerofoil DU 97-W300Mod, Wind Energy, 7, 295-307, 2004.

Timmer, W. and Van Rooij, R.: Summary of the Delft University wind turbine dedicated airfoils, J. Sol. Energ.-T. ASME, 125 , 488-496, 2003.

Torres-Nieves, S., Maldonado, V., Lebrón, J., Kang, H.-S., Meneveau, C., and Castillo, L.: Free-stream turbulence effects on the flow around an S809 wind turbine airfoil, in: Progress in Turbulence and Wind Energy IV, Springer, 275-279, 2012.

Traphan, D., Meinlschmidt, P., Schlüter, F., Lutz, O., Peinke, J., and Gülker, G.: High-speed measurements of different laminarturbulent transition phenomena on rotor blades by means of infrared thermography and stereoscopic PIV, in: 10th Pacific Symposium on Flow Visualization and Image Processing, 2015.

Tropea, C., Yarin, A. L., and Foss, J. F.: Springer handbook of experimental fluid mechanics, vol. 1, Springer Science \& Business Media, 543-551, 2007.

Zhong, S., Chong, T. P., and Hodson, H. P.: A comparison of spreading angles of turbulent wedges in velocity and thermal boundary layers, J. Fluids Eng., 125, 267-274, 2003. 